\title{
Advantages of hydroponics in edible cacti production
}

\begin{abstract}
Hydroponic culture is a method of growing plants using nutrient solution (water and fertilizer) with or without the use of an artificial medium. This system can avoid the costly and time-consuming task of soil sterilization to prevent soil-borne disease and enable precise fertilizer management. Therefore, hydroponic culture provides advantages in the production of edible Opuntia, which is conventionally produced through soil or pot culture. To date, we have studied the effects of hydroponic culture involving the deep flow technique (DFT) on the growth of edible Opuntia, and showed that hydroponics has several advantages compared with the commercially practiced methods. Thus, we think hydroponics may be an ideal method of edible Opuntia cultivation for farmers and horticultural hobbyists based in cities, who typically practice soil and pot culture. In this short review, we describe recent progress and findings on the hydroponic culture of edible Opuntia.
\end{abstract}

Keywords: deep flow technique, drought stress, Edible Opuntia, hydroponics culture, invention, LED, light environment, plant factory, spines
Volume 2 Issue 4 - 2018

\section{Takanori Horibe}

Department of Bioscience and Biotechnology, Chubu University, Japan

Correspondence: Takanori Horibe, Department of Bioscience and Biotechnology, Chubu University, Kasugai, Aichi, 487-8501, Japan, Tel +8I-0568-5 I-9|23, Fax +8I-092-642-29|3, Email t-horibe@isc.chubu.ac.jp

Received: November 18,2017 | Published: July 10, 2018

\section{Introduction}

The stem of the cactus Opuntia (genus Opuntia, subfamily Opuntioideae, family Cactaceae), commonly referred to as the nopal cactus or prickly pear, is a major source of fruit, vegetable and forage in areas, where the soils are poor or are becoming poor and result in very low yield of traditional cultivars. ${ }^{1}$ In some countries, Opuntia species are also used as remedies and folk medicine for a variety of health problems including burns, edema, and indigestion. ${ }^{2,3}$ Edible Opuntia is also produced in Japan, where they are produced mainly in Kasugai City, Aichi Prefecture, although production scale is still small. We are working with Kasugai City to promote production and consumption of edible Opuntia in Japan. Opuntia species are so important commercial crop that cultivation technics which lead to higher production and quality are needed. Opuntia plants are commonly produced through soil or pot culture. Major problems in growing vegetables, including edible Opuntia, using soil are soil-borne disease, salt accumulation, and difficulty in fertilizer management. ${ }^{4}$ In hydroponic culture, plants are grown using nutrient solution (water and fertilizer), with or without the use of an artificial medium. Soilborne disease and weeds are eliminated in hydroponic culture because there is no soil and precise fertilizer management is also possible. ${ }^{4}$ Therefore, hydroponic culture conveys many advantages for edible Opuntia production, and we have shown that edible Opuntia can be grown by simple hydroponic culture. ${ }^{5}$

\section{Discussion}

\section{Application of hydroponics for edible Opuntia production}

Hydroponic culture involves growing plants without soil. This system can avoid the costly and time-consuming task of soil sterilization to prevent soil-borne disease and enable precise fertilizer management. ${ }^{4,6}$ We first investigated the effects of hydroponic culture involving the deep flow technique (DFT) on the growth of edible Opuntia, and its effect on spine development on daughter cladodes. ${ }^{5}$ Then, we showed that edible Opuntia can be grown by DTF and it is effective for reducing number of spines on cladodes. A number of beneficial functions have been ascribed to spines, including participation in zoochorous dispersal ${ }^{7,8}$ mechanical protection from herbivores, ${ }^{7}$ shading of the stem, ${ }^{9}$ reflection of light. ${ }^{10}$ However, spines on cladodes are one of the most undesirable characteristics of edible Opuntia for consumers. Therefore, cultivation method which can suppress spine development lead to improvement of cladode quality. For the hydroponic culture of most vegetables, seeds are spread on the commercially available cultivation panel; however, in the case of edible Opuntia, vegetative propagation using the stem is commonly used for its production because this method is much faster and easier compared with seed propagation. Thus, an appropriate method for the hydroponic culture of edible Opuntia should be developed. We designed a new cost-effective and simple method for the hydroponic culture of edible Opuntia (Figure 1), and showed its effectiveness by comparing the growth of cladodes by this method and by pot culture using a growth chamber and a greenhouse. ${ }^{11}$

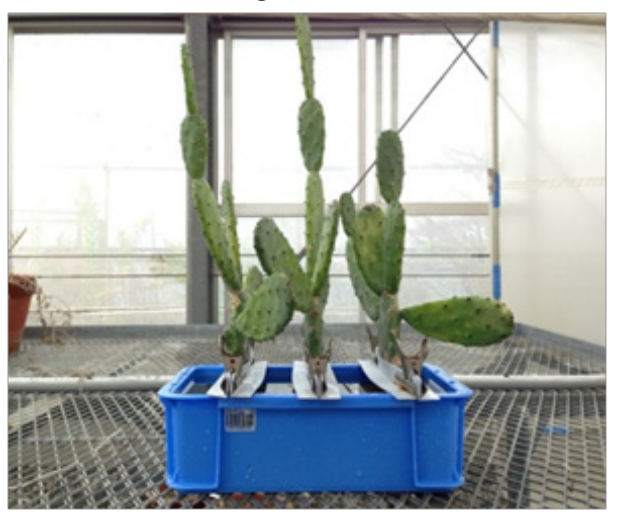

Figure I Hydroponic culture of edible Opuntia.

\section{Edible Opuntia production in plant factory}

Hydroponics is also a basic method for plant production in plant factories. Plant factories can be defined as horticulture greenhouses or automated facilities where vegetables and crops can be produced 
throughout the year controlling environmental conditions, such as light, temperature, humidity, $\mathrm{CO}_{2}$, and nutrient availability by ICT. ${ }^{12,13}$ There are two main types of plant factories. These include artificial lighting-based fully enclosed systems and natural sunlightbased systems. Hydroponic culture is mainly used as the cultivation system in enclosed plant factories, whereas both soil and hydroponic technologies are used in natural sunlight systems. The main advantages of modern plant factories are the food safety and steady supply of food crops to the market. Using artificial lighting-based fully enclosed plant factory, high quality pesticide-free vegetables can be produced throughout the year due to controlled, optimal cultivation environment. ${ }^{14}$ Furthermore, because it does not need sunlight and natural soil, this type of plant factory can be built in any location and in any building.

Hence, because its productivity is not dependent on climate and soil fertility, it enables food production even in regions where agriculture is not feasible. Edible Opuntia has many features suitable

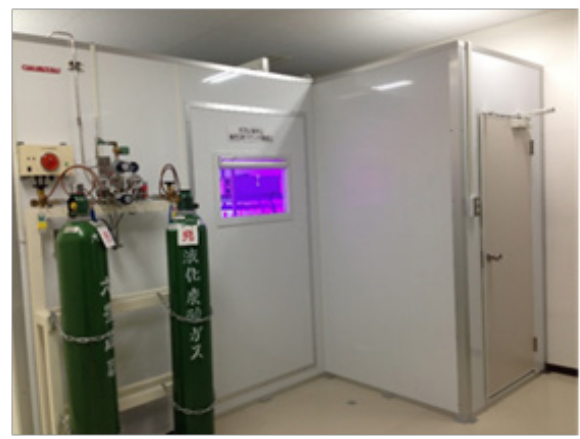

Figure 2 Edible Opuntia production in plant factory.

\section{Conclusion}

Edible cacti are industrially important plants and produced for various purposes. It includes healthy vegetable, fruits, forage, processed foods, and natural dye production. Thus, cultivation techniques which lead to higher production and quality are needed. We have shown that edible cactus can be efficiently cultivated by hydroponic culture with controlled environmental conditions, which can lead to improvement in its quality. Consequently, we propose that hydroponics is powerful tools to cultivate edible cacti and improve their productivity and quality, although more cumulative evidence is necessary.

\section{Acknowledgements}

None.

\section{Conflict of interest}

Author declares that there is no conflict of interest.

\section{References}

1. García Saucedo PA, Valdez Morales M, Valverde ME, et al. Plant regeneration of three Opuntia genotypes used as human food. Plant Cell Tissue Organ Cult. 2005;80(2):215-219.

2. El Mostafa K, El Kharrassi Y, Badreddine A, et al. Nopal cactus (Opuntia ficus-indica) as a source of bioactive compounds for nutrition, health and disease. Molecules. 2014;19(9):14879-14901. for the production in plant factories, which require large running costs. For instance, it grows rapidly and can be vegetatively propagated through stems (in the short term until harvest). Additionally, Opuntia can be planted on cultivation panels at high density (effective use of available space) under relatively low light intensity using artificial light. we have cultivated edible Opuntia in enclosed-type plant factory and investigated the effects of red and blue light emitting diode (LED) light on the growth and spine occurrence in daughter cladodes (Figure 2). We have shown that light wavelength strongly affects the growth and number of spines in daughter cladodes. ${ }^{15}$ Thus, it seems that the growth of edible Opuntia can be manipulated by regulating the environmental conditions, which in turn will be effective for improving the growth and quality of edible cactus. These days, people have strong interest in the safety and health benefits of foods and there are greater demands for high quality, pesticide-free, and functional foods. Therefore, stable production of edible Opuntia with increased nutritive value through environment control in plant factories might be practiced in the near future.

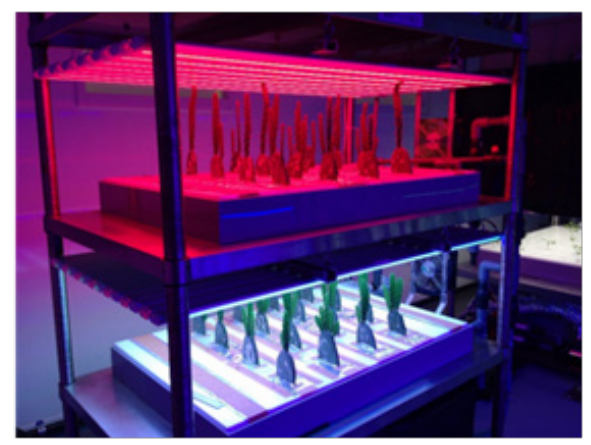

3. Shetty AA, Rana MK, Preetham SP. Cactus: a medicinal food. J Food Sci Technol. 2012;49(5):530-536.

4. Lakkireddy KKR, Kasturi K, Sambasiva RKRS. Role of hydroponics and aeroponics in soilless culture in commercial food production. JAST. 2012;1:26-35

5. Horibe T, Yamada K. Hydroponics culture of edible Opuntia 'Maya': drought stress affects the development of spines on daughter cladodes. Environ Control Biol. 2016;54(3):153-156.

6. Wahome PK, Oseni TO, Masarirambi MT, et al. Effects of different hydroponics systems and growing media on the vegetative growth, yield and cut flower quality of gypsophila (Gypsophila paniculata L.). World J Agric Sci. 2011;7(6):692-698.

7. Bobich EG, Nobel PS. Vegetative reproduction as related to biomechanics, borphology and anatomy of four cholla cactus species in the Sonoran Desert. Ann Bot. 2001;87:485-493.

8. Frego KA, Staniforth RJ. Factors determining the distribution of Opuntia fragilis in the boreal forest of southeastern Manitoba. Can J Bot. 1985;63(12):2377-2382.

9. Norman F, Martin CE. Effects of spine removal on Coryphantha vivipara in central Kansas. Am Midl Nat. 1986;116(1):118-124.

10. Loik ME. The effect of cactus spines on light interception and Photosystem II for three sympatric species of Opuntia from the Mojave Desert. Physiol Plant. 2008;134(1):87-98.

11. Horibe T. A Cost-Effective, Simple, and Productive Method of Hydroponic Culture of Edible Opuntia "Maya". Environ Control Biol. 2017;55(4):171-174. 
12. Hirama J. The history and advanced technology of plant factories Environ Control Biol. 2015;53(2):47-48.

13. Yanata S, Takata K. Plant factory: the possible measures to revitalize the Wakayama's economy, regional studies series no. 43 (Revised Edition). Institute of Economic Research: Wakayama University; 2014:1-22.
14. Yamori W, Zhang G, Takagaki M, et al. Feasibility study of rice growth in plant factories. J Rice Res. 2014;2.

15. Horibe T, Iwagaya Y, Kondo H, et al. Hydroponics Culture of Edible Opuntia 'Maya': Effect of Constant Red and Blue Lights on Daughter Cladodes Growth and Spine Development. Environ Control Biol. 2016;54(4):165-169. 